\title{
Subjective cognitive complaints and neuropsychological test performance following military-related traumatic brain injury
}

\author{
Louis M. French, PsyD; ${ }^{1-2}$ Rael T. Lange, PhD; ${ }^{1,3 *}$ Tracey A. Brickell, D.Psych ${ }^{1-2}$ \\ ${ }^{1}$ Defense and Veterans Brain Injury Center, Bethesda, MD; Neurology, Walter Reed National Military Medical Center, \\ Bethesda, MD; and Center for Neuroscience and Regenerative Medicine, Bethesda, MD; ${ }^{2}$ Uniformed Services University \\ of the Health Sciences, Bethesda, MD; ${ }^{3}$ The University of British Columbia, Vancouver, British Columbia, Canada
}

\begin{abstract}
This study examined the relation between neuropsychological test performance and self-reported cognitive complaints following traumatic brain injury (TBI). Participants were 109 servicemembers from the U.S. military who completed a neuropsychological evaluation within the first 2 yr following mild-severe TBI. Measures included the Personality Assessment Inventory (PAI), Posttraumatic Stress Disorder Symptom Checklist-Civilian version (PCL-C), Neurobehavioral Symptom Inventory (NSI), and 17 select measures from a larger neurocognitive test battery that corresponded to three self-reported cognitive complaints from the NSI (i.e., memory, attention/concentration, and processing speed/organization). Self-reported cognitive complaints were significantly correlated with psychological distress (PCL-C total: $r=0.50-0.58$; half the PAI clinical scales: $r=$ $0.40-0.58$ ). In contrast, self-reported cognitive complaints were not significantly correlated with overall neurocognitive functioning (with the exception of five measures). There was a low rate of agreement between neurocognitive test scores and self-reported cognitive complaints. For the large minority of the sample (38.5\%-45.9\%), self-reported cognitive complaints were reported in the presence of neurocognitive test scores that fell within normal limits. In sum, self-reported cognitive complaints were not associated with neurocognitive test performance, but rather were associated with psychological distress. These results provide information to contextualize cognitive complaints following TBI.
\end{abstract}

Key words: cognitive complaints, military, Neurobehavioral Symptom Inventory, neurocognitive measures, Personality Assessment Inventory, psychological distress, PTSD Checklist, self-reported symptoms, servicemembers, traumatic brain injury.

\section{INTRODUCTION}

Traumatic brain injury (TBI) care, beyond the initial neurological and neurosurgical care and related interventions for more severe injury, is focused on amelioration of self-reported symptoms, modification of environmental factors, and improvement of clinician-observed deficits. This treatment model relies heavily on the participation of the patient in the treatment process. While this is an

\footnotetext{
Abbreviations: $\mathrm{ACT}=$ Auditory Consonant Trigrams, $\mathrm{ANOVA}=$ analysis of variance, CPT-II = Conner's Continuous Performance Test-Second Edition, CVLT-II = California Verbal Learning TestSecond Edition, DOD = Department of Defense, LOC = loss of consciousness, $\mathrm{mTBI}=$ mild traumatic brain injury, NSI $=$ Neurobehavioral Symptom Inventory, PAI = Personality Assessment Inventory, PCL-C = Posttraumatic Stress Disorder Symptom Checklist-Civilian version, $\mathrm{PD}=$ Parkinson disease, $\mathrm{PTA}=$ posttraumatic amnesia, RCFT = Rey Complex Figure Test, SD = standard deviation, TBI = traumatic brain injury, TMT $=$ Trail Making Test, TOL = Tower of London, VA = Department of Veterans Affairs, WAIS-III = Wechsler Adult Intelligence Scale-Third Edition, WMT = Word Memory Test, WNL = within normal limits, WRAMC = Walter Reed Army Medical Center.

*Address all correspondence to Rael T. Lange, PhD; Defense and Veterans Brain Injury Center, Walter Reed National Military Medical Center, Building 8, Room 2264, 8901 Wisconsin Ave, Bethesda, MD 20814; 240-997-5284.

Email: rael.lange@gmail.com, rael.t.lange@us.army.mil http://dx.doi.org/10.1682/JRRD.2013.10.0226
} 
admirable model, it is also fraught with problems (e.g., poor insight, limited communication skills, intimidation by the medical care process, or deliberate misrepresentation), such that limitations of self-reported symptoms could potentially reduce the reliability of this information.

Of particular concern are subjective perceptions of cognitive symptoms. Problems with accuracy in selfreported cognitive symptoms are seen in a variety of health conditions, including cognitive functioning after cancer treatment [1], systemic lupus erythematosus [2], epilepsy [3], multiple sclerosis [4], Parkinson disease (PD) [5], and schizophrenia [6]. This reduced accuracy is generally related to overreporting of symptoms, but varies according to the condition. In some conditions, these difficulties are related to altered cognition or insight or, more commonly, mood changes. In one study with three separate groups (healthy controls given medications, adults with epilepsy on medication, and patients with idiopathic PD) [7], subjective perception of cognitive effects was more related to mood than objective test performance. The medical disability adjudication process itself can cause alterations in accurate self-assessment [8].

It has been recognized for some time that there can be differences between a patient's subjective report of dysfunction and (1) the ratings of a significant other and (2) objective measures of that domain. For example, in a sample of $50 \mathrm{TBI}$ patients with mixed severity (33 severe TBI), patients did not differ in accuracy of self-reported physical problems when compared with ratings by their family caregivers. However, TBI patients consistently underreported cognitive/behavioral problems and emotional symptoms as compared with family responses [9]. It is important to appreciate that the accuracy of familyreported symptoms is also questionable. In a sample of moderate to severe TBI, Lannoo and colleagues found that family ratings of impairment across four domains did not differ from patient reports. However, patient and family reports were also not related to the results of neuropsychological test measurements [10].

Differences between self-reported cognitive complaints and neuropsychological test performance following TBI have also been reported elsewhere. In a sample of patients with mild TBI (mTBI) recruited from a hospital emergency department and followed over a 6 mo period, 39 percent reported subjective cognitive complaints. However, there was no relationship between subjective cognitive complaints and objective neuropsychological test results or the contents of patients' daily logs of functioning. Rather, sub- jective cognitive complaints were more related to poorer physical functioning, emotional distress, and lower educational levels [11]. Emotional distress, particularly mood, has been implicated by a number of other studies as being more influential in subjective reporting of cognitive dysfunction than objective test findings [12-14]. In a sample of veterans with a history of mTBI, self-ratings on a neurobehavioral symptom checklist were compared with the results of a brief neuropsychological examination. Self-ratings of attention and thinking/organization were not significantly correlated with test findings, but were related to increased reporting of psychiatric symptoms [15].

Given the importance of self-report of symptoms in the treatment as well as the compensation and pension process around TBI in the military, we were interested in exploring how accurate patient self-report is in determining the presence and level of cognitive dysfunction in a military TBI population of mixed severity and concurrent polytrauma, a group in which this issue had not been previously explored. Consistent with previous work, we hypothesized that subjective reports of cognitive problems would have limited correlation with objective measures and would instead be more highly related to emotional distress.

\section{METHODS}

\section{Participants}

Participants were 109 male U.S. military servicemembers (89.0\% Caucasian; age: mean \pm standard deviation [SD] $29.3 \pm 8.7$, range 19-56) who sustained a TBI and were evaluated at the Walter Reed Army Medical Center (WRAMC), Washington, DC, following injuries sustained (1) while deployed to Iraq (72.5\%) or Afghanistan (5.5\%) or (2) as a result of other non-combat-related operations/ incidents (22.0\%). The majority of the sample were injured as the result of a blast-related incident (64.2\%) and sustained an mTBI (45.9\%) (moderate TBI $=25.7 \%$, severe TBI $=28.4 \%$ ). Duration of loss of consciousness (LOC) ranged from < $15 \mathrm{~min}$ (56.9\%), 16-59 $\mathrm{min}$ (6.4\%), 1-24 h (6.4\%), to $>1 \mathrm{~d}(15.6 \%)$ (missing $=11.9 \%)$. Duration of posttraumatic amnesia (PTA) ranged from $<24 \mathrm{~h}(49.5 \%)$, 1-7 d (29.4\%), to >7 d (19.3\%) (missing = 1.8\%). Traumarelated intracranial abnormalities were present in almost half the sample (present $=41.3 \%$, absent $=39.4 \%$, no scan $/$ missing $=19.3 \%$ ). Level of education was as follows: General Educational Development/12 yr (44.0\%) and 12+ yr (56.0\%). The mean time tested postinjury was 6.2 mo 
$(\mathrm{SD}=5.3$, range $=1-24 \mathrm{mo})$. Descriptive statistics of demographic and injury-related variables by TBI severity (i.e., mild vs moderate-severe) are presented in Table $\mathbf{1 .}$

\section{Participant Selection and Classification}

Patients were selected from a larger sample of 463 U.S. military servicemembers who had been referred to the TBI clinic at WRAMC for neuropsychological consultation within the first 2 yr postinjury (between February 2002 and January 2009) following a suspected or confirmed TBI. Participants had been referred for a neuropsychological evaluation for a number of reasons, but typically for treatment planning or to aid in decisions about fitness for continued military service. Out of the original
463 participants, individuals were selected for the sample if they met the following criteria: (1) were male (92.7\% of sample); (2) had sustained a closed TBI (100\% of sample); (3) had completed the Personality Assessment Inventory (PAI) (83.4\% of sample) and had a valid PAI profile (71.9\% of those administered); (4) had been administered the Word Memory Test (WMT) (88.6\% of sample) and had passed the WMT using standard cutoffs (64.4\% of those administered); and (5) had completed the Posttraumatic Stress Disorder Symptom Checklist-Civilian version (PCL-C) (80.6\% of sample), Neurobehavioral Symptom Inventory (NSI) (58.5\% of sample), and a core set of neurocognitive measures ( $74.3 \%$ of sample).

Table 1.

Descriptive statistics of demographic and injury-related variables by traumatic brain injury (TBI) severity.

\begin{tabular}{|c|c|c|c|c|}
\hline Variable & Mild TBI & Moderate-Severe TBI & $p$-Value & $d$ \\
\hline Age (yr), Mean \pm SD & $31.0 \pm 9.1$ & $27.8 \pm 8.1$ & 0.06 & 0.37 \\
\hline Time Tested Postinjury (mo), Mean \pm SD & $7.0 \pm 5.6$ & $5.5 \pm 5.0$ & 0.16 & 0.27 \\
\hline Education, $n(\%)$ & & & 0.43 & - \\
\hline GED/12 yr & $20(40.0)$ & 28 (47.5) & & \\
\hline $12+\mathrm{yr}$ & $30(60.0)$ & $31(52.5)$ & & \\
\hline Race, $n(\%)$ & & & 0.76 & - \\
\hline Caucasian & $44(88.0)$ & $53(89.8)$ & & \\
\hline Other & $6(12.0)$ & $6(10.2)$ & & \\
\hline Loss of Consciousness, $n(\%)$ & & & - & - \\
\hline None & $14(28.0)$ & $3(5.1)$ & & \\
\hline $1-15 \min$ & $36(72.0)$ & $12(20.3)$ & & \\
\hline 16-59 min & $0(0)$ & 7 (11.9) & & \\
\hline $1-24 \mathrm{~h}$ & $0(0)$ & 7 (11.9) & & \\
\hline$>1$ to $7 \mathrm{~d}$ & $0(0)$ & $12(20.3)$ & & \\
\hline$>7 \mathrm{~d}$ & $0(0)$ & $5(8.5)$ & & \\
\hline Unknown & $0(0)$ & $13(22.0)$ & & \\
\hline Posttraumatic Amnesia, $n$ (\%) & & & - & - \\
\hline$<1 \min$ & $20(40.0)$ & $0(0)$ & & \\
\hline $1-15 \min$ & $12(24.0)$ & $0(0)$ & & \\
\hline $16-59 \min$ & $12(24.0)$ & $0(0)$ & & \\
\hline $1-24 \mathrm{~h}$ & $5(10.0)$ & $5(8.5)$ & & \\
\hline$>1$ to $7 \mathrm{~d}$ & $0(0)$ & $32(54.2)$ & & \\
\hline$>7$ to $>30 \mathrm{~d}$ & $0(0)$ & $21(35.6)$ & & \\
\hline Unknown & $1(2.0)$ & $1(1.7)$ & & \\
\hline Intracranial Abnormality, $n$ (\%) & & & $<0.001$ & - \\
\hline Absent & $31(62.0)$ & $12(21.8)$ & & \\
\hline Present & $11(22.0)$ & $34(61.8)$ & & \\
\hline No Scan/Missing & $12(24.0)$ & $9(16.4)$ & & \\
\hline Mechanism of Injury, $n$ (\%) & & & 0.45 & - \\
\hline Nonblast & $16(32.0)$ & $23(39.0)$ & & \\
\hline Blast & $34(68.0)$ & $36(61.0)$ & & \\
\hline
\end{tabular}


Diagnosis and classification of TBI severity was undertaken by a number of healthcare professionals, who completed a routine comprehensive clinical evaluation as part of the standard clinical pathway at WRAMC. The large majority of evaluations were completed by an advance practice nurse or physician's assistant trained to evaluate TBI. Some evaluations were also completed by neuropsychologists and social workers trained specifically in TBI evaluations. TBI evaluations typically include (1) medical chart review, (2) patient interview, (3) family interview, and (4) case conferencing with other healthcare professionals. For clinical purposes, diagnosis of TBI is based on the duration of LOC, PTA, and/or alteration of consciousness present immediately postinjury and neuroradiological scans. Self-reported symptoms are routinely obtained during the TBI evaluation but are not used for diagnostic or classification purposes.

Classification of TBI severity was as follows: (1) severe TBI: PTA > 7 d, (2) moderate TBI: PTA $24 \mathrm{~h}$ to $7 \mathrm{~d}$ and LOC $<24$ h, and (3) mTBI: PTA $<24$ h and LOC $<15$ min. For those patients classified in the mTBI group, there are two important issues to note. First, it was our preference to define mTBI based on LOC criterion of $<30 \mathrm{~min}$. However, the available information precluded us from applying this criterion (i.e., available categorical data $=\mathrm{LOC}<15 \mathrm{~min}$ and LOC 16-60 min). Second, inconsistent with the Department of Defense (DOD) clinical guidelines (Management of Concussion/mTBI Working Group, 2009), mTBI was classified when PTA and LOC fell within the mild range and traumarelated intracranial abnormality was present. For the purposes of this study, our preference was to classify these patients as having a "complicated mTBI" (rather than a moderate TBI). The importance of this distinction has been discussed elsewhere [16].

\section{Measures and Procedure}

\section{Psychological Measures}

Psychological measures included the NSI [17], PCL-C [18], and PAI [19]. For this study, only three items from the NSI were included, which measured self-reported cognitive complaints of attention/concentration (item 13: "poor concentration, can't pay attention, easily distracted”), memory (item 14: “forgetfulness, can't remember things"), and processing speed/organization (item 16: "slowed thinking, difficulty getting organized, can't finish things”). The NSI requires the test taker to rate the presence/severity of each symptom on a 5-point scale as follows: $0=$ none, $1=$ mild,
2 = moderate, 3 = severe, and $4=$ very severe. For the PAI, participants were not included if their T-scores exceeded the recommended cutoff on any of the four validity scales (i.e., Inconsistency, Infrequency, Negative Impression, or Positive Impression).

\section{Neurocognitive Measures}

Neurocognitive measures included the (1) Auditory Consonant Trigrams (ACT): 36” Interval Delay [20]; (2) Trail Making Test (TMT): Part A and Part B [21]; (3) Tower of London (TOL): Total Correct, Total Moves, and Total Initiation Time; (4) California Verbal Learning Test-Second Edition (CVLT-II): Total Trials 1-4 and Free Recall Long Delay [22]; (5) Rey Complex Figure Test (RCFT): Copy, Immediate Recall, and Delayed Recall [23]; (6) selected subtests from the Wechsler Adult Intelligence Scale-Third Edition (WAIS-III): Block Design, Digit Symbol-Coding, Letter-Number Sequencing [24]; and (7) Conner's Continuous Performance Test-Second Edition (CPT-II): Omissions, Commissions, and Hit Rate [25]. These neurocognitive tests were selected to include measures that provided an objective assessment of cognitive functioning that would correspond to three self-reported cognitive complaints on the NSI: (1) attention/concentration, (2) memory, and (3) processing speed/organization. In order to evaluate cognitive effort, patients also completed the WMT [26]. Patients were excluded if their scores on the WMT fell below the criterion recommended in the manual.

For this study, three neurocognitive index scores were generated by combining select neurocognitive measures. The measures that were combined to create the three index scores were as follows: (1) attention/concentration index: WAIS-III Letter Number Sequencing; TMT Part A and Part B; ACT 36" Interval Delay; and CPT-II Omissions, Commissions, and Hit Rate; (2) memory index: CVLT-II Total 1-5 and Long Delayed Recall, RCFT Immediate Recall and Delayed Recall, and ACT 36" Interval Delay; and (3) processing speed/organization index: WAIS-III Digit Symbol Coding and Block Design; RCFT Copy; TOL Total Correct, Total Moves, and Total Initiation Time; and TMT Part A and Part B. Note that not all measures included in the index scores were mutually exclusive. Due to the multidimensionality of some tests (e.g., TMT), some measures were included in more than one index score. Before the calculation of the three neurocognitive index scores, raw scores on each test were converted to standard scores (e.g., z-scores, T-scores, scaled scores) as per the instructions in the manual and then 
converted to T-scores (mean $\pm \mathrm{SD}=50 \pm 10$ ) where necessary in order to place all measures on a common scale. The three neurocognitive index scores were then generated by calculating the mean T-score across the selected measures in each index.

\section{RESULTS}

\section{Relation Between Cognitive Complaints and Neuropsychological Measures}

\section{Total Sample}

Pearson correlation coefficients between the three self-reported cognitive complaints and the neuropsychological measures (neurocognitive and psychological) are presented in Table 2. Self-reported cognitive complaints were not significantly correlated with the majority of individual neurocognitive test scores, with the exception of 5 of the 17 measures. For these measures, the strength of the relation was weak ( $r=0.19-0.27)$. In addition, these measures were not consistently associated with corresponding self-reported cognitive complaints. The correlation between the attention/concentration index and self-reported complaints of attention/concentration was very low $(r=-0.06)$. Similarly, the correlation between the processing speed/organization index and self-reported complaints of processing speed/organization was also very low $(r=-0.04)$. The correlation between the memory index and self-reported complaints of memory was statistically significant $(p<0.01)$, but the strength of the relation was weak $(r=0.25)$.

For the psychological measures, all three self-reported cognitive complaints were consistently and significantly correlated with the PCL-C total score $(r=0.50-0.58)$ and more than half the PAI clinical scales. The PAI scales with the strongest relation to all three self-reported cognitive complaints were the Anxiety scale ( $r=0.51-0.56$ ), followed by the Depression ( $r=0.47-0.54)$, Schizophrenia ( $r=0.45-0.56)$, Somatic Complaints $(r=0.40-0.53)$, and Anxiety-Related Disorders ( $r=0.40-0.47)$ scales.

\section{Traumatic Brain Injury Severity Subgroups}

To explore the influence of TBI severity, the sample was divided into two TBI severity subgroups (i.e., 50 mTBI, 59 moderate-severe TBI). Pearson correlation coefficients between the three self-reported cognitive complaints and the neuropsychological measures, by TBI severity subgroups, are presented in Table 3. Self-reported cognitive complaints were again not significantly correlated with the majority of individual neurocognitive test scores in both TBI severity subgroups, with the exception of 5 of the 17 measures in the mTBI subgroup and 1 of 17 measures in the moderate-severe TBI subgroup. However, these measures were not consistently associated with corresponding self-reported cognitive complaints. In the mTBI group, there was a weak association [27] between the three neurocognitive indexes and the corresponding subjective cognitive complaints of attention/concentration ( $r=0.13$ ), memory $(r=-0.27)$, and processing speed/ organization $(r=-0.08)$. In the moderate-severe TBI subgroup, there was a similarly weak association between the neurocognitive indexes and corresponding subjective cognitive complaints of attention/concentration $(r=-0.20)$, memory $(r=-0.22)$, and processing speed/organization $(r=-0.02)$.

For the psychological measures, in both the mTBI and moderate-severe TBI subgroups, all three selfreported cognitive complaints were again consistently and significantly correlated with the PCL-C total score $(r=0.44-0.63)$ and at least half the PAI clinical scales. In the moderate TBI subgroup, the PAI scales with the strongest relation to all three self-reported cognitive complaints were the Anxiety ( $r=0.51-0.57)$, Depression ( $r=$ $0.57-0.60)$, and Schizophrenia $(r=0.58-0.63)$ scales. A similar pattern of results was also found in the mTBI subgroup, though the strength of this relation was lower. In the mTBI subgroup, the PAI scales with the strongest relation to all three self-reported cognitive complaints were the Anxiety ( $r=0.43-0.63)$, Depression $(r=0.34$ 0.53), Schizophrenia ( $r=0.30-0.51$ ), and Somatic Complaints $(r=0.37-0.53)$ scales.

Descriptive statistics and group comparisons for the three neurocognitive indexes and all psychological measures, by TBI severity groups, are presented in Table 4. There were no significant differences between groups for all measures, with the exception of the attention/concentration index ( $p=0.007, d=0.53$ [medium effect size]). Paradoxically, the moderate-severe TBI group had higher scores on the attention/concentration index than the mTBI group.

\section{Rate of Agreement Between Cognitive Complaints and Neurocognitive Performance}

To examine the rate of agreement between self-reported cognitive complaints and neurocognitive test performance, 
JRRD, Volume 51, Number 6, 2014

Table 2.

Relation (Pearson correlation coefficient) between self-reported cognitive complaints and neurocognitive/psychological measures.

\begin{tabular}{|c|c|c|c|c|}
\hline \multirow[b]{2}{*}{ Measure } & \multicolumn{4}{|c|}{ Self-Reported Cognitive Complaint ( $r$ ) } \\
\hline & $\begin{array}{c}\text { Attention/ } \\
\text { Concentration }\end{array}$ & $\begin{array}{l}\text { Forgetfulness/ } \\
\text { Poor Memory }\end{array}$ & $\begin{array}{c}\text { Processing Speed/ } \\
\text { Organization }\end{array}$ & $\begin{array}{c}\text { Total Cognitive } \\
\text { Complaints* }\end{array}$ \\
\hline \multicolumn{5}{|l|}{ Neurocognitive Index } \\
\hline Attention/Concentration & -0.06 & -0.04 & -0.05 & 0.04 \\
\hline Memory & $-0.21^{\dagger}$ & $-0.25^{\ddagger}$ & -0.15 & $-0.19^{\dagger}$ \\
\hline Processing Speed/Organization & 0.01 & -0.12 & -0.04 & -0.01 \\
\hline \multicolumn{5}{|l|}{ Neurocognitive Measure } \\
\hline WAIS-III Letter Number Sequencing & -0.01 & 0.00 & -0.06 & 0.09 \\
\hline TMT Part A & -0.02 & -0.12 & -0.13 & -0.04 \\
\hline TMT Part B & -0.06 & -0.05 & -0.08 & 0.01 \\
\hline ACT 36” Interval Delay & $-0.27^{\ddagger}$ & $-0.25^{\ddagger}$ & $-0.21^{\dagger}$ & $-0.22^{\dagger}$ \\
\hline CPT-II Omissions & 0.11 & $0.19^{\dagger}$ & $0.23^{\dagger}$ & $0.20^{\dagger}$ \\
\hline CPT-II Commissions & -0.01 & 0.17 & 0.03 & 0.04 \\
\hline CPT-II Hit Rate & 0.07 & -0.01 & 0.10 & 0.10 \\
\hline CVLT-II Total 1-5 & -0.07 & -0.14 & -0.08 & -0.15 \\
\hline CVLT-II Long Delayed Recall & -0.16 & $-0.21^{\dagger}$ & -0.10 & $-0.20^{\dagger}$ \\
\hline RCFT Immediate Recall & $-0.21^{\dagger}$ & $-0.22^{\dagger}$ & -0.14 & -0.19 \\
\hline RCFT Delayed Recall & $-0.21^{\dagger}$ & -0.17 & -0.10 & -0.17 \\
\hline WAIS-III Digit Symbol Coding & 0.06 & 0.03 & 0.01 & 0.03 \\
\hline WAIS-III Block Design & 0.06 & 0.02 & 0.14 & 0.12 \\
\hline RCFT Copy & -0.04 & -0.02 & -0.01 & 0.03 \\
\hline TOL Total Correct & 0.06 & -0.05 & 0.00 & -0.01 \\
\hline TOL Total Moves & 0.08 & -0.08 & -0.01 & 0.03 \\
\hline TOL Total Initiation Time & -0.02 & -0.14 & 0.02 & -0.07 \\
\hline \multicolumn{5}{|l|}{ Psychological Measures } \\
\hline PCL-C Total score & $0.50^{\ddagger}$ & $0.58^{\ddagger}$ & $0.52^{\ddagger}$ & $0.51^{\ddagger}$ \\
\hline PAI Somatic Complaints & $0.40^{\ddagger}$ & $0.49^{\ddagger}$ & $0.53^{\ddagger}$ & $0.48^{\ddagger}$ \\
\hline PAI Anxiety & $0.51^{\ddagger}$ & $0.53^{\ddagger}$ & $0.56^{\ddagger}$ & $0.54^{\ddagger}$ \\
\hline PAI Anxiety-Related Disorders & $0.40^{\ddagger}$ & $0.40^{\ddagger}$ & $0.47^{\ddagger}$ & $0.46^{\ddagger}$ \\
\hline PAI Depression & $0.47^{\ddagger}$ & $0.52^{\ddagger}$ & $0.54^{\ddagger}$ & $0.50^{\ddagger}$ \\
\hline PAI Mania & $0.29^{\ddagger}$ & $0.30^{\ddagger}$ & $0.30^{\ddagger}$ & $0.34^{\ddagger}$ \\
\hline PAI Paranoia & $0.24^{\dagger}$ & $0.37^{\ddagger}$ & $0.36^{\ddagger}$ & $0.35^{\ddagger}$ \\
\hline PAI Schizophrenia & $0.45^{\ddagger}$ & $0.53^{\ddagger}$ & $0.56^{\ddagger}$ & $0.53^{\ddagger}$ \\
\hline PAI Borderline Features & $0.34^{\ddagger}$ & $0.33^{\ddagger}$ & $0.37^{\ddagger}$ & $0.38^{\ddagger}$ \\
\hline PAI Antisocial Features & 0.10 & 0.09 & 0.09 & 0.12 \\
\hline PAI Alcohol Problems & 0.05 & 0.05 & 0.01 & 0.02 \\
\hline PAI Drug Problems & 0.03 & 0.06 & 0.05 & 0.04 \\
\hline PAI Aggression & 0.14 & $0.21^{\dagger}$ & 0.18 & $0.22^{\dagger}$ \\
\hline PAI Suicide & -0.01 & 0.05 & 0.08 & 0.05 \\
\hline PAI Stress & $0.27^{\ddagger}$ & $0.35^{\ddagger}$ & $0.31^{\ddagger}$ & $0.33^{\ddagger}$ \\
\hline PAI Nonsupport & $0.25^{\ddagger}$ & $0.25^{\ddagger}$ & $0.27^{\ddagger}$ & $0.26^{\ddagger}$ \\
\hline PAI Treatment Rejection & $-0.39^{\ddagger}$ & $-0.34^{\ddagger}$ & $0.39^{\ddagger}$ & $-0.39^{\ddagger}$ \\
\hline PAI Dominance & -0.03 & -0.04 & -0.06 & -0.01 \\
\hline PAI Warmth & -0.05 & -0.16 & -0.12 & -0.14 \\
\hline
\end{tabular}

Note: $N=109$.

*Number of self-reported cognitive complaints rated as "moderate or higher" on items 13 (attention), 14 (memory), and 16 (thinking/organization) of Neurobehavioral Symptom Inventory. Maximum total symptoms $=3$.

${ }^{\dagger} p<0.05$.

${ }_{p}^{\ddagger} p<0.01$.

ACT = Auditory Consonant Trigrams, CPT = Conner's Continuous Performance Test-Second Edition, CVLT-II = California Verbal Learning Test-Second Edition, PAI = Personality Assessment Inventory, PCL-C = Posttraumatic Stress Disorder Symptom Checklist-Civilian version, RCFT = Rey Complex Figure Test, TMT = Trail Making Test, TOL $=$ Tower of London, WAIS-III $=$ Wechsler Adult Intelligence Scale-Third Edition. 
Table 3.

Relation (Pearson correlation coefficient) between self-reported cognitive complaints and neurocognitive and psychological measures by traumatic brain injury (TBI) severity.

\begin{tabular}{|c|c|c|c|c|c|c|}
\hline \multirow{3}{*}{ Measure } & \multicolumn{6}{|c|}{ Self-Reported Cognitive Complaint (r) } \\
\hline & \multicolumn{2}{|c|}{ Attention/Concentration } & \multicolumn{2}{|c|}{$\begin{array}{l}\text { Forgetfulness/ } \\
\text { Poor Memory }\end{array}$} & \multicolumn{2}{|c|}{$\begin{array}{c}\text { Processing Speed/ } \\
\text { Organization }\end{array}$} \\
\hline & Mild TBI & $\begin{array}{l}\text { Moderate- } \\
\text { Severe TBI }\end{array}$ & Mild TBI & $\begin{array}{l}\text { Moderate- } \\
\text { Severe TBI }\end{array}$ & Mild TBI & $\begin{array}{l}\text { Moderate- } \\
\text { Severe TBI }\end{array}$ \\
\hline \multicolumn{7}{|l|}{ Neurocognitive Index } \\
\hline Attention/Concentration & 0.13 & -0.20 & 0.02 & -0.05 & -0.01 & -0.10 \\
\hline Memory & -0.19 & -0.22 & -0.27 & -0.22 & -0.25 & -0.09 \\
\hline Processing Speed/Organization & -0.05 & 0.04 & -0.14 & -0.11 & -0.08 & -0.02 \\
\hline \multicolumn{7}{|l|}{ Neurocognitive Measure } \\
\hline WAIS-III Letter Number Sequencing & 0.16 & -0.12 & 0.20 & -0.14 & -0.01 & -0.08 \\
\hline TMT Part A & -0.03 & -0.01 & -0.20 & -0.03 & -0.20 & -0.10 \\
\hline TMT Part B & -0.14 & -0.02 & -0.12 & -0.01 & -0.09 & -0.09 \\
\hline ACT 36” Interval Delay & $-0.39^{*}$ & -0.20 & $-0.32^{\dagger}$ & -0.20 & $-0.42^{*}$ & -0.08 \\
\hline CPT-II Omissions & $0.41^{*}$ & -0.09 & $0.34^{\dagger}$ & 0.13 & $0.40^{*}$ & 0.10 \\
\hline CPT-II Commissions & -0.07 & 0.04 & 0.15 & 0.18 & -0.08 & 0.10 \\
\hline CPT-II Hit Rate & 0.27 & -0.09 & 0.08 & -0.08 & 0.28 & -0.04 \\
\hline CVLT-II Total 1-5 & -0.01 & -0.09 & -0.09 & -0.13 & -0.14 & -0.03 \\
\hline CVLT-II Long Delayed Recall & -0.06 & -0.25 & -0.09 & $-0.31^{\dagger}$ & -0.07 & -0.13 \\
\hline RCFT Immediate Recall & -0.23 & -0.20 & $-0.35^{\dagger}$ & -0.12 & -0.26 & -0.07 \\
\hline RCFT Delayed Recall & $-0.31^{\dagger}$ & -0.14 & $-0.30^{\dagger}$ & -0.07 & -0.26 & -0.01 \\
\hline WAIS-III Digit Symbol Coding & 0.01 & 0.10 & -0.02 & 0.05 & -0.14 & 0.12 \\
\hline WAIS-III Block Design & 0.06 & 0.06 & 0.06 & -0.01 & 0.14 & 0.13 \\
\hline RCFT Copy & 0.08 & -0.11 & 0.09 & -0.11 & 0.10 & -0.08 \\
\hline TOL Total Correct & -0.01 & 0.10 & -0.01 & -0.08 & -0.02 & 0.01 \\
\hline TOL Total Moves & -0.06 & 0.15 & -0.13 & -0.04 & -0.16 & 0.07 \\
\hline TOL Total Initiation Time & -0.16 & 0.10 & -0.24 & -0.05 & -0.03 & 0.05 \\
\hline \multicolumn{7}{|l|}{ Psychological Measure } \\
\hline PCL-C Total Score & $0.46^{*}$ & $0.54^{*}$ & $0.62^{*}$ & $0.54^{*}$ & $0.63^{*}$ & $0.44^{*}$ \\
\hline PAI Somatic Complaints & $0.37^{*}$ & $0.41^{*}$ & $0.50^{*}$ & $0.49^{*}$ & $0.53^{*}$ & $0.53^{*}$ \\
\hline PAI Anxiety & $0.43^{*}$ & $0.57^{*}$ & $0.44^{*}$ & $0.60^{*}$ & $0.63^{*}$ & $0.51^{*}$ \\
\hline PAI Anxiety-Related Disorders & $0.35^{\dagger}$ & $0.46^{*}$ & $0.31^{\dagger}$ & $0.46^{*}$ & $0.53^{*}$ & $0.41^{*}$ \\
\hline PAI Depression & $0.34^{\dagger}$ & $0.60^{*}$ & $0.49^{*}$ & $0.57^{*}$ & $0.53^{*}$ & $0.58^{*}$ \\
\hline PAI Mania & 0.20 & $0.38^{*}$ & 0.27 & $0.36^{*}$ & 0.27 & $0.34^{*}$ \\
\hline PAI Paranoia & 0.20 & $0.28^{\dagger}$ & $0.41^{*}$ & $0.34^{*}$ & $0.41^{*}$ & $0.34^{*}$ \\
\hline PAI Schizophrenia & $0.30^{\dagger}$ & $0.58^{*}$ & $0.43^{*}$ & $0.63^{*}$ & $0.51^{*}$ & $0.61^{*}$ \\
\hline PAI Borderline Features & 0.24 & $0.42^{*}$ & 0.27 & $0.40^{*}$ & $0.33^{\dagger}$ & $0.41^{*}$ \\
\hline PAI Antisocial Features & -0.03 & 0.19 & -0.04 & 0.19 & -0.04 & 0.18 \\
\hline PAI Alcohol Problems & 0.09 & 0.03 & 0.09 & 0.02 & 0.11 & -0.04 \\
\hline PAI Drug Problems & -0.01 & 0.06 & 0.14 & -0.01 & 0.14 & -0.03 \\
\hline PAI Aggression & 0.03 & $0.26^{\dagger}$ & 0.14 & $0.28^{\dagger}$ & 0.06 & $0.28^{\dagger}$ \\
\hline PAI Suicide & -0.07 & 0.05 & 0.12 & 0.03 & 0.03 & 0.11 \\
\hline PAI Stress & 0.20 & $0.32^{\dagger}$ & $0.30^{\dagger}$ & $0.41^{*}$ & $0.37^{\dagger}$ & $0.26^{\dagger}$ \\
\hline PAI Nonsupport & $0.33^{\dagger}$ & 0.21 & $0.37^{*}$ & 0.18 & $0.44^{*}$ & 0.12 \\
\hline PAI Treatment Rejection & $-0.32^{\dagger}$ & $-0.45^{*}$ & $-0.36^{\dagger}$ & $-0.33^{\dagger}$ & $-0.43^{*}$ & $-0.35^{*}$ \\
\hline PAI Dominance & -0.10 & 0.01 & -0.10 & 0.01 & -0.09 & -0.03 \\
\hline PAI Warmth & -0.10 & -0.03 & -0.24 & -0.11 & -0.17 & -0.08 \\
\hline
\end{tabular}

Note: $N=109$ (50 mild TBI, 59 moderate-severe TBI).

${ }^{*} p<0.01$.

${ }^{\dagger} p<0.05$.

ACT $=$ Auditory Consonant Trigrams, CPT-II = Conner's Continuous Performance Test-Second Edition, CVLT-II = California Verbal Learning Test-Second Edition, PAI = Personality Assessment Inventory, PCL-C = Posttraumatic Stress Disorder Symptom Checklist-Civilian version, RCFT = Rey Complex Figure Test, TMT $=$ Trail Making Test, TOL $=$ Tower of London, WAIS-III $=$ Wechsler Adult Intelligence Scale-Third Edition . 
individual scores on the three neurocognitive indexes and the three self-reported cognitive complaints were dichotomized to reflect the presence or absence of (1) low scores on each neurocognitive index and (2) clinically elevated cognitive complaints. Low scores on the neurocognitive indexes were defined as mean neurocognitive index T-scores that fell below the 16th percentile. Clinically elevated cognitive complaints were defined as NSI items that were endorsed as moderate or higher.

\section{Total Sample}

Overall, the rate of agreement between self-reported cognitive complaints and corresponding neurocognitive index scores was low. For attention/concentration, (1) 0 per- cent of the sample endorsed self-reported complaints of attention and had a low score on the attention index (i.e., Agreement: Index Low + Compliant Present); (2) 55.0 percent did not endorse attention problems and had scores on the attention index that fell within normal limits (WNL) (i.e., Agreement: Index WNL + Compliant WNL); (3) 45.0 percent had self-reported complaints of attention, but had scores on the attention index that fell WNL (i.e., Disagreement: Index WNL + Compliant Present); and (4) 0 percent of the sample did not endorse attention problems, but had low scores on the attention index (i.e., Disagreement: Index Low + Compliant WNL).

For memory, (1) 6.4 percent of the sample endorsed self-reported complaints of memory and had a low score

Table 4.

Descriptive statistics, group comparisons, and effect sizes for the neurocognitive indexes and psychological measures by traumatic brain injury (TBI) severity.

\begin{tabular}{|c|c|c|c|c|}
\hline \multirow{2}{*}{ Measure } & \multicolumn{2}{|c|}{ Mean \pm SD } & \multirow{2}{*}{$p$-Value } & \multirow{2}{*}{$d$} \\
\hline & Mild TBI & Moderate-Severe TBI & & \\
\hline \multicolumn{5}{|l|}{ Neurocognitive Index } \\
\hline Attention/Concentration & $49.0 \pm 3.8$ & $51.4 \pm 4.9$ & 0.007 & 0.53 \\
\hline Memory & $49.1 \pm 7.6$ & $50.6 \pm 8.0$ & 0.31 & 0.20 \\
\hline Processing Speed/Organization & $50.8 \pm 4.5$ & $50.6 \pm 6.2$ & 0.86 & 0.03 \\
\hline \multicolumn{5}{|l|}{ Psychological Measure } \\
\hline PCL-C Total Score & $36.5 \pm 14.7$ & $33.3 \pm 13.7$ & 0.24 & 0.23 \\
\hline PAI Somatic Complaints & $58.2 \pm 11.1$ & $59.0 \pm 10.0$ & 0.71 & 0.07 \\
\hline PAI Anxiety & $52.3 \pm 11.6$ & $50.7 \pm 9.7$ & 0.44 & 0.15 \\
\hline PAI Anxiety-Related Disorders & $51.6 \pm 11.1$ & $49.0 \pm 9.8$ & 0.20 & 0.25 \\
\hline PAI Depression & $59.1 \pm 14.5$ & $56.4 \pm 11.3$ & 0.29 & 0.21 \\
\hline PAI Mania & $50.6 \pm 10.0$ & $51.4 \pm 9.2$ & 0.67 & 0.08 \\
\hline PAI Paranoia & $54.5 \pm 10.5$ & $53.9 \pm 10.1$ & 0.76 & 0.06 \\
\hline PAI Schizophrenia & $53.6 \pm 13.0$ & $53.3 \pm 10.9$ & 0.89 & 0.03 \\
\hline PAI Borderline Features & $53.6 \pm 11.8$ & $54.2 \pm 11.7$ & 0.80 & 0.05 \\
\hline PAI Antisocial Features & $54.9 \pm 9.6$ & $56.8 \pm 10.6$ & 0.33 & 0.19 \\
\hline PAI Alcohol Problems & $49.8 \pm 8.0$ & $49.5 \pm 7.5$ & 0.85 & 0.04 \\
\hline PAI Drug Problems & $48.6 \pm 6.5$ & $48.7 \pm 6.8$ & 0.88 & 0.03 \\
\hline PAI Aggression & $57.7 \pm 13.7$ & $55.7 \pm 13.3$ & 0.44 & 0.15 \\
\hline PAI Suicide & $46.7 \pm 5.8$ & $47.3 \pm 4.8$ & 0.58 & 0.11 \\
\hline PAI Stress & $53.9 \pm 10.9$ & $55.5 \pm 10.9$ & 0.45 & 0.15 \\
\hline PAI Nonsupport & $50.6 \pm 11.8$ & $51.6 \pm 10.6$ & 0.66 & 0.08 \\
\hline PAI Treatment Rejection & $52.5 \pm 9.7$ & $52.1 \pm 8.9$ & 0.81 & 0.05 \\
\hline PAI Dominance & $54.8 \pm 8.9$ & $54.2 \pm 9.8$ & 0.72 & 0.07 \\
\hline PAI Warmth & $46.5 \pm 12.5$ & $46.0 \pm 11.3$ & 0.83 & 0.04 \\
\hline
\end{tabular}

Note: $N=109$ (50 mild TBI, 59 moderate-severe TBI).

PAI = Personality Assessment Inventory, PCL-C = Posttraumatic Stress Disorder Symptom Checklist-Civilian, SD = standard deviation. 
on the memory index (i.e., Agreement: Index Low + Compliant Present); (2) 45.0 percent did not endorse memory problems and had scores on the memory index that fell WNL (i.e., Agreement: Index WNL + Compliant WNL); (3) 45.9 percent had self-reported complaints of memory, but had scores on the memory index that fell WNL (i.e., Disagreement: Index WNL + Compliant Present); and (4) 2.8 percent of the sample did not endorse memory problems, but had low scores on the memory index (i.e., Disagreement: Index Low + Compliant WNL).

For processing speed/organization, (1) 2.8 percent of the sample endorsed self-reported complaints of processing speed/organization and had a low score on the processing speed/organization index (i.e., Agreement: Index Low + Compliant Present); (2) 57.8 percent did not endorse processing speed/organization problems and had scores on the processing speed/organization index that fell WNL (i.e., Agreement: Index WNL + Compliant WNL); (3) 38.5 percent had self-reported complaints of processing speed/organization, but had scores on the processing speed/organization index that fell WNL (i.e., Disagreement: Index WNL + Compliant Present); and (4) 0.9 percent of the sample did not endorse processing speed/ organization problems, but had low scores on the processing speed/organization index (i.e., Disagreement: Index Low + Compliant WNL).

\section{Traumatic Brain Injury Severity Subgroups}

A similar pattern of results was also found when stratifying by TBI severity, with a low rate of agreement between self-reported cognitive complaints and corresponding neurocognitive index scores found in both the mTBI and moderate-severe TBI subgroups. In addition, there were no significant differences $(p>0.05)$ in the rates of agreement between the mTBI and moderatesevere TBI subgroups for all comparisons. For example, 0 percent of both the mTBI subgroup and moderatesevere TBI subgroup endorsed self-reported complaints of attention/concentration and had a low score on the attention index. Similarly, there were no appreciable differences in the percentage of each subgroup that had selfreported cognitive complaints of attention/concentration in the presence of objective measures of attention/concentration that fell WNL (46.0\% mTBI vs $44.1 \%$ moderate-severe TBI). Data are not shown but can be provided on request.

\section{Influence of Number of Cognitive Complaints on Psychological Measures}

\section{Total Sample}

To explore the influence of the number of self-reported cognitive complaints on the psychological measures, we divided the sample into two groups based on the number of self-reported cognitive complaints endorsed as moderate or higher: (1) 0 cognitive complaints $(n=44)$ and (2) 1-3 cognitive complaints $(n=65)$. Descriptive statistics, group comparisons (analysis of variance [ANOVA]), and effect sizes [28] for the PCL-C total score and the 18 PAI scales, by group, are presented in Table 5 .

There were statistically significant differences between groups for the PCL-C total score ( $d=1.01$ [very large effect size]) and for 13 of the 18 PAI scales $(d=0.42$ [medium effect size] to $d=1.11$ [very large effect size]). On these measures, those participants who endorsed 1-3 cognitive complaints had statistically significantly higher scores on the PCL-C and the majority of the PAI scales. For two of the PAI scales (Treatment Rejection and Warmth), those participants who endorsed 1-3 self-reported cognitive complaints had statistically significantly lower scores.

\section{Traumatic Brain Injury Severity Subgroups}

Each of the two TBI severity subgroups were also divided into two groups based on the number of selfreported cognitive complaints endorsed as moderate or higher: mTBI (0 cognitive complaints [ $n=19], 1-3$ cognitive complaints [ $n=31])$; moderate-severe TBI ( 0 cognitive complaints [ $n=25], 1-3$ cognitive complaints [ $n=34]$ ). Descriptive statistics, group comparisons (ANOVA), and effect sizes [28] for the PCL-C total score and the 18 PAI scales, by group, for each of the TBI severity subgroups separately, are presented in Table 6.

In the mTBI subgroup, there were statistically significant differences between groups for the PCL-C total score ( $d=0.96$ [very large effect size]) and for 6 of the 18 PAI scales $(d=0.64-0.97)$. Although not significantly different (likely due to small sample sizes), medium effect sizes were also found on an additional five PAI scales ( $d=$ $0.40-0.57)$. On these measures, those participants who endorsed 1-3 self-reported cognitive complaints had higher scores on the PCL-C and the majority of the PAI scales. For two of the PAI scales (Treatment Rejection and Dominance), those participants who endorsed 1-3 selfreported cognitive complaints had lower scores. 
Table 5.

Comparison of mean \pm standard deviation scores on Posttraumatic Stress Disorder Symptom Checklist-Civilian version (PCL-C) and Personality Assessment Inventory and (PAI) scales by dichotomous self-reported complaint groups.

\begin{tabular}{|c|c|c|c|c|}
\hline Measure & $\begin{array}{l}0 \text { Cognitive Complaints } \\
\qquad(n=44)\end{array}$ & $\begin{array}{l}\text { 1-3 Cognitive Complaints } \\
\qquad(n=65)\end{array}$ & $p$-Value & $d$ \\
\hline$\overline{\text { PCL-C Total Score }}$ & $27.3 \pm 9.2$ & $39.9 \pm 14.7$ & $<0.001$ & 1.01 \\
\hline PAI Somatic Complaints & $53.2 \pm 7.9$ & $62.4 \pm 10.5$ & $<0.001$ & 0.98 \\
\hline PAI Anxiety & $46.3 \pm 7.9$ & $54.9 \pm 10.8$ & $<0.001$ & 0.88 \\
\hline PAI Anxiety-Related Disorder & $45.6 \pm 9.0$ & $53.3 \pm 10.3$ & $<0.001$ & 0.79 \\
\hline PAI Depression & $51.6 \pm 10.8$ & $61.7 \pm 12.6$ & $<0.001$ & 0.86 \\
\hline PAI Mania & $48.1 \pm 9.6$ & $53.0 \pm 9.0$ & 0.007 & 0.54 \\
\hline PAI Paranoia & $50.6 \pm 8.8$ & $56.6 \pm 10.6$ & 0.002 & 0.61 \\
\hline PAI Schizophrenia & $46.6 \pm 8.9$ & $58.1 \pm 11.4$ & $<0.001$ & 1.11 \\
\hline PAI Borderline Features & $49.1 \pm 9.1$ & $57.2 \pm 12.1$ & $<0.001$ & 0.75 \\
\hline PAI Antisocial Features & $54.3 \pm 8.8$ & $57.0 \pm 10.9$ & 0.17 & 0.27 \\
\hline PAI Alcohol Problems & $49.3 \pm 7.6$ & $49.9 \pm 7.8$ & 0.68 & 0.08 \\
\hline PAI Drug Problems & $49.0 \pm 7.0$ & $48.5 \pm 6.4$ & 0.70 & 0.07 \\
\hline PAI Aggression & $53.3 \pm 12.1$ & $58.8 \pm 14.0$ & 0.04 & 0.42 \\
\hline PAI Suicide & $47.2 \pm 5.5$ & $47.0 \pm 5.2$ & 0.85 & 0.04 \\
\hline PAI Stress & $51.1 \pm 9.7$ & $57.3 \pm 11.0$ & 0.003 & 0.59 \\
\hline PAI Nonsupport & $47.6 \pm 9.6$ & $53.5 \pm 11.5$ & 0.006 & 0.55 \\
\hline PAI Treatment Rejection & $55.9 \pm 8.1$ & $49.8 \pm 9.2$ & 0.001 & 0.70 \\
\hline PAI Dominance & $55.6 \pm 8.9$ & $53.6 \pm 9.6$ & 0.28 & 0.21 \\
\hline PAI Warmth & $49.1 \pm 10.8$ & $44.3 \pm 12.1$ & 0.04 & 0.42 \\
\hline
\end{tabular}

In the moderate-severe TBI subgroup, there were statistically significant differences between groups for the PCL-C total score $(d=1.04$ [very large effect size]) and for 12 of the 18 PAI scales $(d=0.54-1.70)$. Although not significantly different (likely due to small sample sizes), medium effect sizes were also found on an additional two PAI scales ( $d=0.45-0.47)$. On these measures, those participants who endorsed 1-3 self-reported cognitive complaints had higher scores on the PCL-C and the majority of the PAI scales. For two of the PAI scales (Treatment Rejection and Warmth), those participants who endorsed 1-3 self-reported cognitive complaints had lower scores.

\section{DISCUSSION}

In general, and as expected, self-reported cognitive complaints were not significantly correlated with the majority of individual neurocognitive test measures. For those measures that were significantly correlated, the relation was weak. Looking at specific domains, memory had the most measures that were significantly correlated with memory complaints, but here, too, the strength of the relation was weak. In every domain, the percentage of people who had accurately self-assessed a deficit was dwarfed by those who claimed deficits in the face of normal performance. Also as expected, all three self-reported cognitive complaints were consistently and significantly correlated with stress symptoms and multiple PAI scales, most notably anxiety and depression. Individuals who had cognitive complaints consistently scored higher on measures of psychological distress. Previous work in a military population has consistently shown the role of emotional distress in symptom reporting after TBI [29-32]. This adds to that body of literature. Perhaps of greater interest, however, is the reason for the mismatch. Diminished insight because of damage to cortical structures that support self-appraisal and monitoring is one possibility. Certainly, these more anterior regions of the brain tend to be more differentially affected in TBI [33]. This possibility is less likely in the patients with milder TBI, but becomes increasingly possible as severity increases. However, contrary to expectations, we found no appreciable differences in the relation 
Table 6.

Comparison of mean scores on the Posttraumatic Stress Disorder Symptom Checklist-Civilian (PCL-C) and Personality Assessment Inventory and (PAI) scales by dichotomous self-reported complaint groups and traumatic brain injury (TBI) severity.

\begin{tabular}{|c|c|c|c|c|c|c|c|c|}
\hline \multirow[b]{3}{*}{ Measure } & \multicolumn{4}{|c|}{ Mild TBI } & \multicolumn{4}{|c|}{ Moderate-Severe TBI } \\
\hline & \multicolumn{2}{|c|}{ Mean \pm SD } & \multirow[b]{2}{*}{$p$-Value } & \multirow[b]{2}{*}{$d$} & \multicolumn{2}{|c|}{ Mean \pm SD } & \multirow[b]{2}{*}{$p$-Value } & \multirow[b]{2}{*}{$d$} \\
\hline & $\begin{array}{c}\text { o Cognitive } \\
\text { Complaints } \\
\quad(n=19)\end{array}$ & $\begin{array}{c}\text { 1-3 Cognitive } \\
\text { Complaints } \\
(n=31)\end{array}$ & & & $\begin{array}{l}\text { 0 Cognitive } \\
\text { Complaints } \\
\quad(n=25)\end{array}$ & $\begin{array}{c}\text { 1-3 Cognitive } \\
\text { Complaints } \\
(n=34)\end{array}$ & & \\
\hline$\overline{\text { PCLC Total }}$ & $28.7 \pm 10.2$ & $41.3 \pm 8.7$ & 0.002 & 0.96 & $26.2 \pm 8.3$ & $38.6 \pm 14.6$ & $<0.001$ & 1.04 \\
\hline PAI Somatic Complaints & $52.2 \pm 7.9$ & $61.9 \pm 6.0$ & 0.002 & 0.97 & $53.9 \pm 8.0$ & $62.8 \pm 9.8$ & $<0.001$ & 0.98 \\
\hline PAI Depression & $53.6 \pm 12.1$ & $62.4 \pm 11.9$ & 0.04 & 0.64 & $50.0 \pm 9.6$ & $61.1 \pm 10.1$ & $<0.001$ & 1.11 \\
\hline PAI Mania & $48.1 \pm 10.8$ & $52.1 \pm 10.2$ & 0.17 & 0.41 & $48.0 \pm 8.7$ & $53.9 \pm 8.8$ & 0.02 & 0.66 \\
\hline PAI Paranoia & $52.6 \pm 10.2$ & $55.7 \pm 15.0$ & 0.33 & 0.29 & $49.1 \pm 7.4$ & $57.5 \pm 10.5$ & 0.001 & 0.91 \\
\hline PAI Schizophrenia & $48.4 \pm 11.1$ & $56.8 \pm 9.2$ & 0.03 & 0.67 & $45.2 \pm 6.7$ & $59.2 \pm 9.5$ & $<0.001$ & 1.70 \\
\hline PAI Borderline Features & $50.1 \pm 10.9$ & $55.8 \pm 10.7$ & 0.10 & 0.49 & $48.3 \pm 7.6$ & $58.6 \pm 12.3$ & $<0.001$ & 1.00 \\
\hline PAI Antisocial Features & $54.7 \pm 8.4$ & $55.0 \pm 13.2$ & 0.90 & 0.04 & $54.0 \pm 9.3$ & $58.9 \pm 11.1$ & 0.08 & 0.47 \\
\hline PAI Suicide & $47.4 \pm 6.8$ & $46.4 \pm 6.8$ & 0.56 & 0.17 & $47.0 \pm 4.3$ & $47.5 \pm 5.1$ & 0.68 & 0.11 \\
\hline PAI Stress & $50.4 \pm 8.7$ & $56.1 \pm 13.9$ & 0.07 & 0.55 & $51.7 \pm 10.5$ & $58.4 \pm 10.6$ & 0.02 & 0.63 \\
\hline PAI Nonsupport & $46.6 \pm 10.3$ & $53.1 \pm 5.2$ & 0.06 & 0.57 & $48.4 \pm 9.2$ & $53.9 \pm 11.1$ & 0.05 & 0.54 \\
\hline PAI Treatment Rejection & $57.2 \pm 8.8$ & $49.6 \pm 11.6$ & 0.007 & 0.83 & $55.0 \pm 7.6$ & $49.9 \pm 9.2$ & 0.03 & 0.59 \\
\hline PAI Dominance & $56.9 \pm 7.0$ & $53.5 \pm 12.2$ & 0.18 & 0.40 & $54.6 \pm 10.2$ & $53.8 \pm 9.7$ & 0.75 & 0.09 \\
\hline PAI Warmth & $49.4 \pm 12.5$ & $44.7 \pm 9.3$ & 0.20 & 0.38 & $48.8 \pm 9.6$ & $43.9 \pm 12.1$ & 0.10 & 0.45 \\
\hline
\end{tabular}

between self-reported cognitive complaints and objective neurocognitive test performance in mTBI versus moderate-severe TBI subgroups.

Another possibility is deliberate misrepresentation. This is an issue in all cases in which there may be external incentives to look worse than one is objectively [34] and certainly an issue in military and Department of Veterans Affairs (VA) cases [35]. In our sample, we do not have data on external incentives. However, all subjects included passed formal measures of effort on cognitive testing and validity measures on personality testing, suggesting that, in at least some domains, they showed adequate levels of validity. It is also possible that the participants' report was a more valid assessment of their everyday function than was the neuropsychological test measures employed. While we know that such measures have limitations [36], we also know that neuropsychological assessment in general [37] is a strong predictor of daily functioning after TBI. We, unfortunately, did not have collateral information on daily functioning in these individuals. Traditional markers of everyday cognitive functioning, such as success at competitive employment, were not applicable in this population of injured servicemembers who remained "employed" in the military but with typically few duties beyond participation in medical treatment.

It is also possible that other clinical conditions may be accounting for subjective cognitive complaints. It is well known that various clinical groups (e.g., psychiatric disorders, personal injury claimants, chronic pain, posttraumatic stress disorder, and soft-tissue injuries) [38-47] and even healthy adults [48-55] often report cognitive symptoms in the absence of brain injury. Finally, we cannot exclude the effects of expectancy. With increased awareness around the occurrence of concussion and TBI, there is also increased awareness around potential long-term effects. This may be especially pronounced in mTBI, where individuals may expect to have persistent problems even though they are showing a good recovery [51,56]. The DOD and VA have developed extensive screening and education programs 
around TBI for all servicemembers, but not without a potential cost around stigma, misattribution, or other negative effects [57-58]. In one study of veterans who screened positive for TBI [59], "friends in the military" was the most frequently cited source of information about TBI and its effects, with the internet being the second highest. This heightens the possibility of misinformation about TBI and its possible consequences. This is unfortunate, because structured, accurate educational interventions have been shown to be effective in reducing postconcussive symptoms in both acute and more chronic TBI in Active Duty servicemembers, veterans, and civilians [60]. Cognitive behavioral interventions that focus on identifying misattributions can be very effective in reducing longer term morbidity in some cases of mTBI [61-63]. Administration of diagnostic tests and sharing of test results with a patient can actually reduce symptom complaints with subsequent reduction in healthcare utilization [64].

\section{STUDY LIMITATIONS}

It is important to recognize that there is some inherent ambiguity in these particular constructs. For example, while reduced processing speed may be a symptom of TBI, it may also reflect subjective cognitive slowing associated with depression; complaints of concentration difficulties may also reflect mood changes or stress symptoms. We do not know what these subjects believe to be the etiology of their cognitive concerns. Strong attribution of symptoms to TBI can contribute to symptom complaints [65].

This study has several methodological limitations that warrant mention. First, we excluded participants from the final sample for poor cognitive effort based on a single symptom validity measure (i.e., WMT). While the WMT is considered to be a clinically valid and reliable measure, current clinical guidelines recommend using more than one symptom validity measure for the purposes of detecting poor cognitive effort [66-67]. It is possible that we included some people who had been misidentified as providing adequate effort when, in fact, they provided inadequate effort. However, it is important to note that we also excluded people if they were identified as providing exaggerated symptom reporting based on the PAI validity scales. The relation between symptom exaggeration and poor cognitive effort is well established [68-79]. As such, the combination of the WMT and PAI validity scales together greatly reduces the likelihood of misidentification and is unlikely to have affected the overall results. Second, no information was available regarding compensation status or external incentives in this sample. External incentives are common in this population. Some servicemembers are motivated to avoid being deployed again or to obtain a disability pension or other financial incentive. This information was not available, and we could not evaluate the influence of external incentives on test performance. Third, no information was available regarding history of previous TBI. Fourth, we relied on a single item to measure each of the cognitive complaints. It is uncertain whether a comprehensive evaluation of these cognitive complaints would result in different conclusions. Fifth, we acknowledge that we compared neuropsychological test performance against population-based normative data rather than change in an individual's performance against his or her own baseline. In some cases, the patient's report of a cognitive change may represent a true decline in his or her own functioning, which was not readily discernible with cognitive testing. In that circumstance, self-appraisal may be more accurate than was suggested here. Given that normative data were age and education adjusted (and in some cases also sex and race adjusted), this likelihood is relatively small but should be recognized. Sixth, the military population might not be reliably generalizable to the larger civilian TBI population, because of patient demographics, typically method of injury, rates of comorbid stress, or other factors. For example, in our sample, we saw strong neurocognitive performance in our moderate/ severe TBI patients. This is consistent with some of our previous work [80] with this population. It may reflect unusual resiliency and recovery as a result of preinjury fitness and health, relatively young age, lack of substance misuse, or other factors in this employed (by definition) group. Finally, although not considered a limitation per se, it is important to acknowledge that the TBI severity classification system used here is not consistent with DOD clinical guidelines. As such, it is important for clinicians who use DOD clinical guidelines to view these results within the context of the classification system used in this study. For example, the mTBI classifications used here include the "mTBI" DOD classification and some individuals who would be considered moderate TBI (i.e., complicated mTBI). 


\section{CONCLUSIONS}

These findings help to contextualize the role of cognitive symptom complaints in this population and underscore the importance of understanding emotional and environmental factors in interpreting subjective concerns. Further, we wish to suggest the value of educational interventions in this population. While not investigated in this study, we believe that a discussion around the meaning of neuropsychological, neuroimaging, and other test results coupled with potential implications for the recovery process and the future is important. Promoting an expectancy of recovery in mild cases and realistically discussing functional implications in more severe cases, both with the patient and the family, may serve to more closely align objective and subjective differences in assessment. Where appropriate, psychiatric contributors to symptoms should be identified and mental health services provided as needed, whether to deal with adjustment issues related to coping with injury, deployment-related stress, overt depression, posttraumatic stress disorder, or other diagnoses.

\section{ACKNOWLEDGMENTS}

\author{
Author Contributions: \\ Study concept and design: L. M. French, R. T. Lange, T. A. Brickell. \\ Acquisition of data: L. M. French, R. T. Lange. \\ Analysis and interpretation of data: L. M. French, R. T. Lange, \\ T. A. Brickell. \\ Drafting of manuscript: L. M. French, R. T. Lange. \\ Critical revision of manuscript for important intellectual content: \\ L. M. French, R. T. Lange, T. A. Brickell. \\ Statistical analysis: R. T. Lange.
}

Financial Disclosures: The authors have declared that no competing interests exist.

Funding/Support: This material was unfunded at the time of manuscript preparation.

Institutional Review: The protocols under which these data were collected were approved by the Institutional Review Board of WRAMC/ Walter Reed National Military Medical Center and in accordance with the guidelines of the Helsinki Declaration. All participants provided informed consent.

Participant Follow-Up: The authors do not plan to inform participants of the publication of this study.

Disclaimer: Portions of these data were presented at the International Neuropsychological Society annual conference, February 2012, Montreal, Quebec City, Canada. The views expressed in this article are those of the authors and do not reflect the official policy of the DOD, VA, or U.S. Government.

\section{REFERENCES}

1. Poppelreuter M, Weis J, Külz AK, Tucha O, Lange KW, Bartsch HH. Cognitive dysfunction and subjective complaints of cancer patients. A cross-sectional study in a cancer rehabilitation centre. Eur J Cancer. 2004;40(1):43-49. [PMID:14687788] http://dx.doi.org/10.1016/j.ejca.2003.08.001

2. Vogel A, Bhattacharya S, Larsen JL, Jacobsen S. Do subjective cognitive complaints correlate with cognitive impairment in systemic lupus erythematosus? A Danish outpatient study. Lupus. 2011;20(1):35-43. [PMID:21233148] http://dx.doi.org/10.1177/0961203310382430

3. Liik M, Vahter L, Gross-Paju K, Haldre S. Subjective complaints compared to the results of neuropsychological assessment in patients with epilepsy: The influence of comorbid depression. Epilepsy Res. 2009;84(2-3):194-200.

[PMID:19264450]

http://dx.doi.org/10.1016/j.eplepsyres.2009.02.006

4. Kinsinger SW, Lattie E, Mohr DC. Relationship between depression, fatigue, subjective cognitive impairment, and objective neuropsychological functioning in patients with multiple sclerosis. Neuropsychology. 2010;24(5):573-80. [PMID:20804245] http://dx.doi.org/10.1037/a0019222

5. Dujardin K, Duhamel A, Delliaux M, Thomas-Antérion C, Destée A, Defebvre L. Cognitive complaints in Parkinson's disease: Its relationship with objective cognitive decline. J Neurol. 2010;257(1):79-84. [PMID:19690907] http://dx.doi.org/10.1007/s00415-009-5268-2

6. Johnson I, Tabbane K, Dellagi L, Kebir O. Self-perceived cognitive functioning does not correlate with objective measures of cognition in schizophrenia. Compr Psychiatry. 2011;52(6):688-92. [PMID:21296346] http://dx.doi.org/10.1016/j.comppsych.2010.12.008

7. Marino SE, Meador KJ, Loring DW, Okun MS, Fernandez HH, Fessler AJ, Kustra RP, Miller JM, Ray PG, Roy A, Schoenberg MR, Vahle VJ, Werz MA. Subjective perception of cognition is related to mood and not performance. Epilepsy Behav. 2009;14(3):459-64. [PMID:19130899] http://dx.doi.org/10.1016/j.yebeh.2008.12.007

8. Greve KW, Bianchini KJ, Brewer ST. The assessment of performance and self-report validity in persons claiming painrelated disability. Clin Neuropsychol. 2013;27(1):108-37. [PMID:23157188] http://dx.doi.org/10.1080/13854046.2012.739646

9. Sbordone RJ, Seyranian GD, Ruff RM. Are the subjective complaints of traumatically brain injured patients reliable? Brain Inj. 1998;12(6):505-15. [PMID:9638327] http://dx.doi.org/10.1080/026990598122467

10. Lannoo E, Colardyn F, Vandekerckhove T, De Deyne C, De Soete G, Jannes C. Subjective complaints versus neuropsychological test performance after moderate to severe head 
injury. Acta Neurochir (Wien). 1998;140(3):245-53. [PMID:9638261]

http://dx.doi.org/10.1007/s007010050091

11. Stulemeijer M, Vos PE, Bleijenberg G, van der Werf SP. Cognitive complaints after mild traumatic brain injury: Things are not always what they seem. J Psychosom Res. 2007;63(6):637-45. [PMID:18061755] http://dx.doi.org/10.1016/j.jpsychores.2007.06.023

12. Chamelian L, Feinstein A. The effect of major depression on subjective and objective cognitive deficits in mild to moderate traumatic brain injury. J Neuropsychiatry Clin Neurosci. 2006;18(1):33-38. [PMID:16525068] http://dx.doi.org/10.1176/appi.neuropsych.18.1.33

13. Gass CS, Apple C. Cognitive complaints in closed-head injury: Relationship to memory test performance and emotional disturbance. J Clin Exp Neuropsychol. 1997;19(2): 290-99. [PMID:9240487] http://dx.doi.org/10.1080/01688639708403858

14. Satz P, Forney DL, Zaucha K, Asarnow RR, Light R, McCleary C, Levin H, Kelly D, Bergsneider M, Hovda D, Martin N, Namerow N, Becker D. Depression, cognition, and functional correlates of recovery outcome after traumatic brain injury. Brain Inj. 1998;12(7):537-53.

[PMID:9653518]

http://dx.doi.org/10.1080/026990598122313

15. Spencer RJ, Drag LL, Walker SJ, Bieliauskas LA. Selfreported cognitive symptoms following mild traumatic brain injury are poorly associated with neuropsychological performance in OIF/OEF veterans. J Rehabil Res Dev. 2010;47(6):521-30. [PMID:20848365] http://dx.doi.org/10.1682/JRRD.2009.11.0181

16. Iverson GL, Lange RT, Gaetz M, Zasler N. Mild traumatic brain injury. In: Zasler N, Katz D, Zafonte R, editors. Brain injury medicine: Principle and practice. New York (NY): Demos Medical Publishing, Inc; 2007. p. 333-71.

17. Cicerone KD, Kalmar K. Persistent postconcussion syndrome: The structure of subjective complaints after mild traumatic brain injury. J Head Trauma Rehabil. 1995;10:1-7. http://dx.doi.org/10.1097/00001199-199510030-00002

18. Weathers FW, Litz BT, Herman DS, Huska JA, Keane TM. PTSD Checklist-Civilian version. Boston (MA): National Center for PTSD, Behavioral Science Division; 1994.

19. Morey LC. Personality assessment inventory: Professional manual. Odessa (FL): Psychological Assessment Resources; 1991.

20. Spreen O, Strauss E. A compendium of neuropsychological tests: Administration, norms, and commentary. 2nd ed. New York (NY): Oxford University Press; 1998.

21. Reitan RM. Trail Making Test: Manual for administration and scoring. South Tucson (AZ): Reitan Neuropsychological Laboratory; 1992.
22. Delis DC, Kramer JH, Kaplan E, Ober BA. The California Verbal Learning Test. 2nd ed. New York (NY): The Psychological Corporation; 2000.

23. Meyers JE, Meyers KR. Rey Complex Figure Test and Recognition Trial: Professional manual. Lutz (FL): Psychological Assessment Resources; 1995.

24. Wechsler D. Wechsler Adult Intelligence Scale. 3rd ed. San Antonio (TX): The Psychological Corporation; 1997.

25. Conners K. Conner's Continuous Performance Test. 2nd ed. Toronto (Canada): Multi-Health Systems Inc; 2002.

26. Green P. Green's Word Memory Test: User manual. Edmonton (Canada): Green's Publishing; 2003.

27. Taylor R. Interpretation of the correlation coefficient: A basic review. J Diagn Med Sonography. 1990;6:35-39. http://dx.doi.org/10.1177/875647939000600106

28. Cohen J. Statistical power analysis for the behavioral sciences. 2nd ed. Hillsdale (NJ): Lawrence Erlbaum Associates; 1988.

29. Belanger HG, Kretzmer T, Vanderploeg RD, French LM. Symptom complaints following combat-related traumatic brain injury: Relationship to traumatic brain injury severity and posttraumatic stress disorder. J Int Neuropsychol Soc. 2010;16(1):194-99. [PMID:19758488] http://dx.doi.org/10.1017/S1355617708090036

30. Lange RT, Brickell T, French LM, Ivins B, Bhagwat A, Pancholi S, Iverson GL. Risk factors for postconcussion symptom reporting after traumatic brain injury in U.S. military service members. J Neurotrauma. 2013;30(4):237-46. [PMID:23126461] http://dx.doi.org/10.1089/neu.2012.2685

31. Schneiderman AI, Braver ER, Kang HK. Understanding sequelae of injury mechanisms and mild traumatic brain injury incurred during the conflicts in Iraq and Afghanistan: Persistent postconcussive symptoms and posttraumatic stress disorder. Am J Epidemiol. 2008;167(12): 1446-52. [PMID:18424429] http://dx.doi.org/10.1093/aje/kwn068

32. Hoge CW, McGurk D, Thomas JL, Cox AL, Engel CC, Castro CA. Mild traumatic brain injury in U.S. Soldiers returning from Iraq. N Engl J Med. 2008;358(5):453-63. [PMID:18234750] http://dx.doi.org/10.1056/NEJMoa072972

33. Lux WE. A neuropsychiatric perspective on traumatic brain injury. J Rehabil Res Dev. 2007;44(7):951-62. [PMID:18075952] http://dx.doi.org/10.1682/JRRD.2007.01.0009

34. Bianchini KJ, Curtis KL, Greve KW. Compensation and malingering in traumatic brain injury: A dose-response relationship? Clin Neuropsychol. 2006;20(4):831-47. [PMID:16980265] http://dx.doi.org/10.1080/13854040600875203 
35. Worthen MD, Moering RG. A practical guide to conducting VA compensation and pension exams for PTSD and other mental disorders. Psychol Inj Law. 2011;4(3-4):187-216. http://dx.doi.org/10.1007/s12207-011-9115-2

36. Donovan NJ, Heaton SC, Kimberg CI, Wen PS, Waid-Ebbs JK, Coster W, Singletary F, Velozo CA. Conceptualizing functional cognition in traumatic brain injury rehabilitation. Brain Inj. 2011;25(4):348-64. [PMID:21323413] http://dx.doi.org/10.3109/02699052.2011.556105

37. Chaytor N, Temkin N, Machamer J, Dikmen S. The ecological validity of neuropsychological assessment and the role of depressive symptoms in moderate to severe traumatic brain injury. J Int Neuropsychol Soc. 2007;13(3): 377-85. [PMID:17445285] http://dx.doi.org/10.1017/S1355617707070592

38. Fox DD, Lees-Haley PR, Earnest K, Dolezal-Wood S. Post-concussive symptoms: Base rates and etiology in psychiatric patients. Clin Neuropsychol. 1995;9(1):89-92. http://dx.doi.org/10.1080/13854049508402064

39. Lees-Haley PR, Brown RS. Neuropsychological complaint base rates of 170 personal injury claimants. Arch Clin Neuropsychol. 1993;8(3):203-9. [PMID:14589632]

http://dx.doi.org/10.1093/arclin/8.3.203

40. Dunn JT, Lees-Haley PR, Brown RS, Williams CW, English LT. Neurotoxic complaint base rates of personal injury claimants: Implications for neuropsychological assessment. J Clin Psychol. 1995;51(4):577-84. [PMID:7593681] http://dx.doi.org/10.1002/1097-4679(199507)51:4<577::AIDJCLP2270510418>3.0.CO;2-E

41. Foa EB, Cashman L, Jaycox L, Perry K. The validation of a self-report measure of posttraumatic stress disorder: The Posttraumatic Diagnostic Scale. Psychol Assess. 1997; 9(4):445-51. http://dx.doi.org/10.1037/1040-3590.9.4.445

42. Mickeviciene D, Schrader H, Obelieniene D, Surkiene D, Kunickas R, Stovner LJ, Sand T. A controlled prospective inception cohort study on the post-concussion syndrome outside the medicolegal context. Eur J Neurol. 2004;11(6): 411-19. [PMID:15171738] http://dx.doi.org/10.1111/j.1468-1331.2004.00816.x

43. Smith-Seemiller L, Fow NR, Kant R, Franzen MD. Presence of post-concussion syndrome symptoms in patients with chronic pain vs mild traumatic brain injury. Brain Inj. 2003;17(3):199-206. [PMID:12623496]

http://dx.doi.org/10.1080/0269905021000030823

44. Radanov BP, Dvorák J, Valach L. Cognitive deficits in patients after soft tissue injury of the cervical spine. Spine. 1992;17(2):127-31. [PMID:1553581] http://dx.doi.org/10.1097/00007632-199202000-00001

45. Iverson GL, McCracken LM. 'Postconcussive' symptoms in persons with chronic pain. Brain Inj. 1997;11(11):783-90. [PMID:9354255] http://dx.doi.org/10.1080/026990597122990
46. Gasquoine PG. Postconcussional symptoms in chronic back pain. Appl Neuropsychol. 2000;7(2):83-89.

[PMID:10863602] http://dx.doi.org/10.1207/S15324826AN0702 3

47. Meares S, Shores EA, Taylor AJ, Batchelor J, Bryant RA, Baguley IJ, Chapman J, Gurka J, Dawson K, Capon L, Marosszeky JE. Mild traumatic brain injury does not predict acute postconcussion syndrome. J Neurol Neurosurg Psychiatry. 2008;79(3):300-306. [PMID:17702772] http://dx.doi.org/10.1136/jnnp.2007.126565

48. Gouvier WD, Uddo-Crane M, Brown LM. Base rates of postconcussional symptoms. Arch Clin Neuropsychol. 1988; 3(3):273-78. [PMID:14589697] http://dx.doi.org/10.1016/0887-6177(88)90019-4

49. Machulda MM, Bergquist TF, Ito V, Chew S. Relationship between stress, coping, and postconcussion symptoms in a healthy adult population. Arch Clin Neuropsychol. 1998; 13(5):415-24. [PMID:14590606] http://dx.doi.org/10.1093/arclin/13.5.415

50. Iverson GL, Lange RT. Examination of "postconcussionlike” symptoms in a healthy sample. Appl Neuropsychol. 2003;10(3):137-44. [PMID:12890639] http://dx.doi.org/10.1207/S15324826AN1003 02

51. Mittenberg W, DiGiulio DV, Perrin S, Bass AE. Symptoms following mild head injury: Expectation as aetiology. J Neurol Neurosurg Psychiatry. 1992;55(3):200-204.

[PMID:1564481] http://dx.doi.org/10.1136/jnnp.55.3.200

52. Trahan DE, Ross CE, Trahan SL. Relationships among postconcussional-type symptoms, depression, and anxiety in neurologically normal young adults and victims of mild brain injury. Arch Clin Neuropsychol. 2001;16(5):435-45. [PMID:14590158] http://dx.doi.org/10.1016/S0887-6177(00)00051-2

53. Sawchyn JM, Brulot MM, Strauss E. Note on the use of the Postconcussion Syndrome Checklist. Arch Clin Neuropsychol. 2000;15(1):1-8. [PMID:14590562]

http://dx.doi.org/10.1093/arclin/15.1.1

54. Wong JL, Regennitter RP, Barrios F. Base rate and simulated symptoms of mild head injury among normals. Arch Clin Neuropsychol. 1994;9(5):411-25. [PMID:14589656] http://dx.doi.org/10.1093/arclin/9.5.411

55. Wang Y, Chan RC, Deng Y. Examination of postconcussion-like symptoms in healthy university students: Relationships to subjective and objective neuropsychological function performance. Arch Clin Neuropsychol. 2006; 21(4):339-47. [PMID:16765018] http://dx.doi.org/10.1016/j.acn.2006.03.006

56. Suhr JA, Gunstad J. Further exploration of the effect of "diagnosis threat" on cognitive performance in individuals with mild head injury. J Int Neuropsychol Soc. 2005;11(1): 23-29. [PMID:15686605] http://dx.doi.org/10.1017/S1355617705050010 
57. Scholten J, Cernich A, Hurley RA, Helmick K. Department of Veterans Affairs's traumatic brain injury screening and evaluation program: Promoting individualized interdisciplinary care for symptomatic veterans. J Head Trauma Rehabil. 2013;28(3):219-22. [PMID:23661074] http://dx.doi.org/10.1097/HTR.0b013e318291daca

58. Logan BW, Goldman S, Zola M, Mackey A. Concussive brain injury in the military: September 2001 to the present. Behav Sci Law. 2013;31(6):803-13. [PMID:24130079] http://dx.doi.org/10.1002/bsl.2092

59. Spencer RJ, McGuire AP, Tree HA, Waldron-Perrine B, Pangilinan PH, Bieliauskas LA. Report of traumatic brain injury information sources among OIF/OEF Veterans undergoing polytrauma evaluations. J Rehabil Res Dev. 2013;50(1):1-6. [PMID:23516078] http://dx.doi.org/10.1682/JRRD.2012.01.0018

60. King EG, Kretzmer TS, Vanderploeg RD, Asmussen SB, Clement VL, Belanger HG. Pilot of a novel intervention for postconcussive symptoms in active duty, veterans, and civilians. Rehabil Psychol. 2013;58(3):272-79.

[PMID:23815304]

http://dx.doi.org/10.1037/a0033116

61. Mittenberg W, Canyock EM, Condit D, Patton C. Treatment of post-concussion syndrome following mild head injury. J Clin Exp Neuropsychol. 2001;23(6):829-36.

[PMID:11910547]

http://dx.doi.org/10.1076/jcen.23.6.829.1022

62. Miller LJ, Mittenberg W. Brief cognitive behavioral interventions in mild traumatic brain injury. Appl Neuropsychol. 1998;5(4):172-83. [PMID:16318443]

http://dx.doi.org/10.1207/s15324826an0504_2

63. Mittenberg W, Tremont G, Zielinski RE, Fichera S, Rayls KR. Cognitive-behavioral prevention of postconcussion syndrome. Arch Clin Neuropsychol. 1996;11(2):139-45. [PMID:14588914] http://dx.doi.org/10.1016/0887-6177(95)00006-2

64. Howard L, Wessely S, Leese M, Page L, McCrone P, Husain K, Tong J, Dowson A. Are investigations anxiolytic or anxiogenic? A randomised controlled trial of neuroimaging to provide reassurance in chronic daily headache. $\mathrm{J}$ Neurol Neurosurg Psychiatry. 2005;76(11):1558-64. [PMID:16227551] http://dx.doi.org/10.1136/jnnp.2004.057851

65. Larson EB, Kondiles BR, Starr CR, Zollman FS. Postconcussive complaints, cognition, symptom attribution and effort among veterans. J Int Neuropsychol Soc. 2013;19(1): 88-95. [PMID:23146625]

66. Heilbronner RL, Sweet JJ, Morgan JE, Larrabee GJ, Millis SR; Conference Participants. American Academy of Clinical Neuropsychology Consensus Conference Statement on the neuropsychological assessment of effort, response bias, and malingering. Clin Neuropsychol. 2009;23(7):1093-1129.

\section{[PMID:19735055]}

http://dx.doi.org/10.1080/13854040903155063

67. Bush SS, Ruff RM, Tröster AI, Barth JT, Koffler SP, Pliskin NH, Reynolds CR, Silver CH. Symptom validity assessment: Practice issues and medical necessity NAN policy \& planning committee. Arch Clin Neuropsychol. 2005;20(4):419-26. [PMID:15896556] http://dx.doi.org/10.1016/j.acn.2005.02.002

68. Boone KB, Lu PH. Impact of somatoform symptomatology on credibility of cognitive performance. Clin Neuropsychol. 1999;13(4):414-19. [PMID:10806453]

http://dx.doi.org/10.1076/1385-4046(199911)13:04;1-Y;FT414

69. Gervais RO, Ben-Porath YS, Wygant DB, Green P. Differential sensitivity of the Response Bias Scale (RBS) and MMPI-2 validity scales to memory complaints. Clin Neuropsychol. 2008;22(6):1061-79. [PMID:18609315] http://dx.doi.org/10.1080/13854040701756930

70. Gervais RO, Rohling ML, Green P, Ford W. A comparison of WMT, CARB, and TOMM failure rates in non-head injury disability claimants. Arch Clin Neuropsychol. 2004; 19(4):475-87. [PMID:15163449] http://dx.doi.org/10.1016/j.acn.2003.05.001

71. Henry GK, Heilbronner RL, Mittenberg W, Enders C, Domboski K. Comparison of the MMPI-2 restructured Demoralization Scale, Depression Scale, and Malingered Mood Disorder Scale in identifying non-credible symptom reporting in personal injury litigants and disability claimants. Clin Neuropsychol. 2009;23(1):153-66.

[PMID:18609325] http://dx.doi.org/10.1080/13854040801969524

72. Iverson GL, Lange RT, Brooks BL, Rennison VL. "Good old days" bias following mild traumatic brain injury. Clin Neuropsychol. 2010;24(1):17-37. [PMID:19830628] http://dx.doi.org/10.1080/13854040903190797

73. Larrabee GJ. Exaggerated MMPI-2 symptom report in personal injury litigants with malingered neurocognitive deficit. Arch Clin Neuropsychol. 2003;18(6):673-86. [PMID:14591440] http://dx.doi.org/10.1016/S0887-6177(02)00157-9

74. Larrabee GJ. Exaggerated pain report in litigants with malingered neurocognitive dysfunction. Clin Neuropsychol. 2003;17(3):395-401. [PMID:14704890] http://dx.doi.org/10.1076/clin.17.3.395.18087

75. Suhr J, Hammers D, Dobbins-Buckland K, Zimak E, Hughes $C$. The relationship of malingering test failure to self-reported symptoms and neuropsychological findings in adults referred for ADHD evaluation. Arch Clin Neuropsychol. 2008;23(5):521-30. [PMID:18562158] http://dx.doi.org/10.1016/j.acn.2008.05.003

76. Suhr J, Tranel D, Wefel J, Barrash J. Memory performance after head injury: Contributions of malingering, litigation status, psychological factors, and medication use. J Clin 
FRENCH et al. Subjective cognitive complaints

Exp Neuropsychol. 1997;19(4):500-514. [PMID:9342686] http://dx.doi.org/10.1080/01688639708403740

77. Tsanadis J, Montoya E, Millis SR, Hanks RA, Fichtenberg NL, Axelrod BN. A negative impression management scale for the Postconcussive Syndrome Questionnaire. J Int Neuropsychol Soc. 2007;13(S1):196-97.

78. Tsanadis J, Montoya E, Hanks RA, Millis SR, Fichtenberg NL, Axelrod BN. Brain injury severity, litigation status, and self-report of postconcussive symptoms. Clin Neuropsychol. 2008;22(6):1080-92. [PMID:18609319] http://dx.doi.org/10.1080/13854040701796928

79. Wygant DB, Sellbom M, Ben-Porath YS, Stafford KP, Freeman DB, Heilbronner RL. The relation between symptom validity testing and MMPI-2 scores as a function of forensic evaluation context. Arch Clin Neuropsychol. 2007;22(4):489-99. [PMID:17350796] http://dx.doi.org/10.1016/j.acn.2007.01.027

80. Lange RT, Brickell TA, French LM, Merritt VC, Bhagwat A, Pancholi S, Iverson GL. Neuropsychological outcome from uncomplicated mild, complicated mild, and moderate traumatic brain injury in US military personnel. Arch Clin Neuropsychol. 2012;27(5):480-94. [PMID:22766317] http://dx.doi.org/10.1093/arclin/acs059

Submitted for publication October 22, 2013. Accepted in revised form March 13, 2014.

This article and any supplementary material should be cited as follows:

French LM, Lange RT, Brickell TA. Subjective cognitive complaints and neuropsychological test performance following military-related traumatic brain injury. J Rehabil Res Dev. 2014;51(6):933-50.

http://dx.doi.org/10.1682/JRRD.2013.10.0226

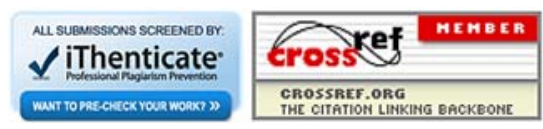


